BMJ Nutrition,

Prevention \& Health

\section{Consumption of industrial processed foods and risk of premenopausal breast cancer among Latin American women: the PRECAMA study}

To cite: Romieu I, Khandpur $\mathrm{N}$ Katsikari A, et al. Consumption of industrial processed foods and risk of premenopausal breast cancer among Latin American women: the PRECAMA study. BMJ Nutrition, Prevention \& Health 2022;0:e000335. doi:10.1136/ bmjnph-2021-000335

- Additional supplemental material is published online only. To view, please visit the journal online (http://dx.doi.org/10. 1136/bmjnph-2021-000335).

For numbered affiliations see end of article.

Correspondence to Dr Sabina Rinaldi, International Agency for Research on Cancer, Lyon, France; rinaldis@iarc.fr

Received 9 July 2021 Accepted 8 December 2021

Check for updates

(C) Author(s) (or their employer(s)) 2022. Re-use permitted under CC BY-NC. No commercial re-use. See rights and permissions. Published by BMJ.

\section{ABSTRACT}

Ultra-processed food intake has been linked to an increased risk of breast cancer in Western populations. No data are available in the Latin American population although the consumption of ultra-processed foods is increasing rapidly in this region.

We evaluated the association of ultra-processed food intake to breast cancer risk in a case-control study including 525 cases (women aged 20-45 years) and 525 matched population-based controls from Chile, Colombia, Costa Rica and Mexico. The degree of processing of foods was classified according to the NOVA classification. Overall, the major contributors to ultra-processed food intake were ready-to-eat/heat foods (18.2\%), cakes and desserts $(16.7 \%)$, carbonated and industrial fruit juice beverages $(16.7 \%)$, breakfast cereals $(12.9 \%)$, sausages and reconstituted meat products (12.1\%), industrial bread $(6.1 \%)$, dairy products and derivatives $(7.6 \%)$ and package savoury snacks $(6.1 \%)$. Ultra-processed food intake was positively associated with the risk of breast cancer in adjusted models ( $0 \mathrm{R}_{\mathrm{T}-\mathrm{T} 1}=1.93 ; 95 \% \mathrm{Cl}=1.11$ to 3.35 ). Specifically, a higher risk was observed with oestrogen receptor positive breast cancer $\left(\mathrm{OR}_{\mathrm{T} \text { - } \mathrm{T}}=2.44,(95 \%\right.$ $\mathrm{Cl}=1.01$ to 5.90 , P-trend $=0.049$ ), while no significant association was observed with oestrogen receptor negative breast cancer $\left(\mathrm{OR}_{\mathrm{T}-\mathrm{T} 1}=1.87,95 \% \mathrm{Cl}=0.43\right.$ to 8.13, P-trend=0.36).

Our findings suggest that the consumption of ultraprocessed foods might increase the risk of breast cancer in young women in Latin America. Further studies should confirm these findings and disentangle specific mechanisms relating ultra-processed food intake and carcinogenic processes in the breast.

\section{INTRODUCTION}

Risk factors for breast cancer in premenopausal women are not well understood. ${ }^{1}$ This may be related to different tumour biology in young women compared with older women. ${ }^{2}$ In Latin America close to $27 \%$ of

\section{What this paper adds}

- This study reports for the first time that the consumption of ultraprocessed foods is associated with an increased risk of breast cancer in young women from Latin American countries.

- Our findings, if confirmed, will be instrumental to support public policies.

breast cancer occurs between 20 and 45 years and increasing trends have been observed in the last decades with a larger proportion of oestrogen receptor negative (ER-) and triple negative (TN) tumours than observed in Western countries. ${ }^{1}$ This population is currently undergoing a rapid lifestyle and nutritional transition switching from a varied traditional diet (including corn tortillas, corn flour cakes, beans and other legumes, soup, homemade stew, vegetable, whole fruit) towards a more homogenous diet rich in industrial ultra-processed foods. ${ }^{34}$

Ultra-processed foods refer to formulations of substances that have been extracted or refined from whole foods through processes like baking, frying, extruding, moulding, reshaping, hydrogenation and hydrolysis. The final food or beverage product created is durable, convenient and palatable, readyto-heat or ready-to-eat as snacks or to replace freshly prepared food-based dishes and meals. ${ }^{5}$ Ultra-processed foods include mostly carbonated soft drinks, sweet and savoury snacks, breakfast cereals, packaged bread, confectionary, cakes and pastries, ice cream, cookies, fruit and vegetables juices, energy drinks, pre-prepared meat, margarine and 
spreads sauces and ready meals. ${ }^{6}$ A recent report from the WHO/Pan American Health Organisation ${ }^{7}$ pointed out the importance of this nutritional change, highlighting a $43 \%$ increase in the sales of ultra-processed foods and drinks in Latin America between 2000 and 2013 while the increase in North America during the same period was only $3 \%$. The fastest increases were observed in Mexico and Chile, while in Costa Rica and Colombia the increase was lower. In 2013, the annual retail sales of ultra-processed foods and drink products in kilograms per capita were 307 in the USA (the world highest), 210 in Mexico (fourth), 202 in Chile (seventh), 120 in Costa Rica and 87 in Colombia. ${ }^{7}$

Classification of foods by degree of food processing could improve our understanding of how dietary factors may relate to chronic diseases. ${ }^{8}$ The NOVA classification categorises food items in accordance to their level of processing into minimally processed, processed culinary ingredients, processed foods and ultra-processed food and drink products. ${ }^{5}$ Ultra-processed foods are often energy dense, rich in saturated and trans-fatty acids, added sugar and salt and low content in fibres and vitamins and have been associated with an increased risk of several chronic diseases, in particular obesity, metabolic syndrome and dyslipidaemia ${ }^{6-11}$ as well as cancer. ${ }^{11}$ Possible mechanisms for these associations may include industrial trans-fatty acids, formed when fats and oils are partly hydrogenated during industrial processing, and that have been linked to the risk of breast and ovarian cancer. ${ }^{10}$ To date there is no study on the potential role of ultra-processed food intake on the risk of cancer in Latin America.

Given the potential for preventive measures, there is an urgent need to investigate the impact of ultra-processed foods on the incidence of cancer, and principally breast cancer, the most common cancer among women in Latin America. ${ }^{9}$

In this manuscript, we report the association of ultraprocessed food intake and risk of breast cancer, overall and by hormonal receptors status, within the framework of the PRECAMA project, an ongoing multicentric population-based case-control study coordinated by the International Agency for Research on Cancer (IARC).

\section{METHODS}

\section{The PRECAMA study}

The PRECAMA study involves scientific teams in four Latin American countries (Chile, Colombia, Costa Rica and Mexico). The methodology has previously been reported. ${ }^{12}$ Through IARC and a central laboratory (the Porter Lab) at the Fred Hutchinson Cancer Research Center (FHCRC) in Seattle, the research teams have harmonised and standardised the protocol approved by at least one Institutional Review Board within each country and by the IARC Ethics Committee.

\section{Selection of cases and controls}

Women with incident primary invasive breast cancer cases were recruited from major cancer hospitals in four Latin American countries before any therapies. ${ }^{12}$ The inclusion criteria were: age $20-45$ years; being resident for $\geq 3$ years in the same city district; having an incident primary invasive breast cancer with positive biopsy and clinical staging according to TNM standards and having menstruated at least once in the past 12 months. To date 525 primary invasive breast cancer incident cases have been recruited among premenopausal women (40 in Chile, 129 in Colombia, 43 in Costa Rica and 313 in Mexico).

Controls (one per case) were selected from the population residing in the same city district as the case for at least 3 years, applying the same exclusion criteria as for the cases, and matched to cases on age ( \pm 3 years), area of residence and healthcare institution membership. Recruitment of cases and controls started in 2012.

\section{Exposure assessment}

During the recruitment visit, trained nurses administered health and lifestyle questionnaires as well as validated and standardised food frequency questionnaires to assess diet. Anthropometry was measured according to standardised protocols and following Lohman's recommendations. ${ }^{13}$ Biological samples (fasting blood and urine) were also collected for biomarker analyses.

\section{Dietary intake assessment}

Dietary intake was assessed through a standardised semiquantitative food frequency questionnaire with countryspecific food list and a standard portion size for each food item. These questionnaires have been validated in women residing in Mexico City ${ }^{14}{ }^{15}$ and in Costa Rica. ${ }^{16}$ In each country, the list of food items was carefully reviewed with input from local nutritionists, and additional foods were added if necessary, according to local nutrition surveys, in order to capture the local diet. Thus, the number of food and drink items in each questionnaire varied across countries, and included 195, 217, 168 and 191 items, respectively, for Chile, Colombia, Costa Rica and Mexico.

For each food item, a commonly used unit or portion size was indicated (specified serving size: a slice, a glass or a natural unit such as one apple), and participants were asked how often, on average, over the previous year, they consumed the specified amount of each food, choosing among 10 frequencies of consumption: $>6$ times/day, 4-5 times/day, 2-3 times/day, 1 time/day, 5-6 times/ week, 2-4 times/week, 1 time/week, 2-3 times/month, $<1$ time/month or never. In addition, information was collected on intake of alcoholic and non-alcoholic drinks (sugar-sweetened beverages, tea, coffee and water), as well as on added sugar, added salt, fat used for cooking and intake of fat from meat. Food items were linked to their macronutrient and micronutrient profile and total caloric intake was calculated based on context-specific food composition tables. 


\section{Categorisation of dietary intake NOVA classification}

Dietary data from the semi-quantitative food frequency questionnaire were used to develop the NOVA classification based on the work by Monteiro et $a l^{5}$ Foods were categorised into four groups according to their degree of processing as: (1) unprocessed or minimally processed foods or NOVA 1 (eg, fresh fruits and vegetables, unprocessed meat, fish...); (2) processed culinary ingredients or NOVA 2 (eg, oils, butter, sugar...); (3) processed foods or NOVA 3 (eg, canned fish, cheeses, fresh bread...); and (4) ultra-processed foods or NOVA 4 (eg, soft drinks, sweet and savoury packaged snacks, processed meat, ready-to-eat/heat foods (burgers, hot dogs, instant soups,....)...). The classification of the various food items in the different NOVA categories was reviewed by local nutritionists in each country (considering the degree of processing of each food item in their local context) and classified in collaboration with Dr Khandpur (University of Sao Paulo, Brazil). In case of doubt between two NOVA categories (3.8\% of the foods), the food item was allocated to both groups while using a percentage in each group (eg, baked custard 30\% NOVA $1 \%$ and $70 \%$ NOVA 4) based on the dietary habits provided by local nutritionists.

This study focuses primarily on identifying and classifying ultra-processed foods (NOVA 4). Ultra-processed foods were classified into 14 subgroups including carbonated beverages and industrial fruit juices, flavoured yoghurt and dairy products, distilled alcoholic beverages, industrial breads, breakfast cereals, confectionery, package sweet snacks, packaged savoury snacks, cakes and desserts, sausage and reconstituted meat products, ready-to-eat/heat foods, industrial cheese and cheese substitutes, margarine and butter substitutes and other ultra-processed foods (including sauces, gravies, jam, mayonnaise, artificial sweeteners).

\section{Pathology review and immunohistochemistry analyses}

Prepared histology sections from tumour biopsy obtained prior to any treatment were reviewed for diagnosis, tumour grade, lymphovascular invasion and stromal and lymphocyte response. Testing was conducted by immunohistochemistry (IHC) for ER (SP1, LabVision, Fremont California), PgR (PgR 636, DAKO, Denmark), human epidermal growth factor receptor 2 (AO485, DAKO, Denmark), according to standardised and optimised protocols that included antigen retrieval when required. All analyses were conducted centrally at the Porter Lab at the FHCRC. Each centre applies standardised protocols for specimen collection. ${ }^{12}$

\section{Laboratory analysis of trans-fatty acids}

Industrial trans-fatty acids blood concentration was measured in a subsample $(\mathrm{n}=284)$ in order to evaluate the correlation with ultra-processed food intake. The methodology has been previously described. ${ }^{10}$ Samples from cases and controls were processed in the same batch, and laboratory staff was blinded to any participant characteristics. The relative amount of each fatty acid, expressed as per cent of total fatty acids, was quantified by integrating the area under the peak and dividing the result by the total area. Coefficients of variation for fatty acids ranged from $1.81 \%$ for large peaks to $9.75 \%$ for the smallest peaks.

\section{Statistical analyses}

Descriptive statistics (median/p10-p90) and proportions were calculated overall and stratified by tumour subtypes considering oestrogen receptors positive (ER+), ER- and TN tumour classification. Matching variables included: age \pm 3 years, country, place of residence and health system membership. Wilcoxon tests were used to compare main characteristics and ultra-processed food intake (kcal) between cases and controls. ${ }^{17}$ Conditional logistic regression was used to calculate OR and $95 \%$ CI for the association of ultra-processed foods intake and breast cancer overall and by receptor status adjusting for potential confounding factors. The quantity of energy (kcal) of ultra-processed foods intake was modelled as tertiles based on the distribution of the controls. Tests for trend were calculated based on the scores of the tertiles. Stratified analyses were conducted on ER + and ER-, and TN tumours and compared with controls groups. Our final multivariate models were adjusted for factors that changed our risk estimate by more than $10 \%$ including education level (proven to be a good marker of overall socio-economic status in Latin American countries classified as $<=$ primary school, secondary school, $>$ secondary school), age at first full-term pregnancy (nulliparous $/<20$, $20-<25,>25$ years), number of full-term pregnancy (continuous), breast feeding (yes/no), physical activity (in hours per day of moderate intensity physical activity) body mass index (BMI) (continuous) and total caloric intake (continuous) (model 1). Further adjustment was conducted by adding to model 1 the quantity of energy from other nova groups (NOVA 1, NOVA 2 and NOVA 3) (model 2) as independent variables. This adjustment was used to further control the potential confounding effect of caloric intake from other food items than ultraprocessed foods. These analyses were repeated using the percentage of total energy intake from ultra-processed foods as sensitivity analyses. The consumption of ultraprocessed foods subgroups was compared in cases and controls and logarithmic transformation was used to normalise skewed variables. Thereafter, the association between ultra-processed food subgroups and breast cancer was explored in multivariate conditional regression models using the Benjamini-Hochberg correction to control for multiple comparisons. ${ }^{18}$

Partial Spearman correlation between blood levels of total industrial trans-fatty acids resulting from industrial processing (in percentage of total fatty acids) and ultraprocess food intake (in grams) was calculated among the 142 cases and 142 controls with available data (joining data from cases and controls to increase sample size) adjusting for case/control status and laboratory batches. 
Further sensitivity analyses were conducted including only ultra-processed food items with potential high content of industrial trans-fatty acids (no detailed food composition was available to identify individual content in trans-fatty acids isomers). Two-sided $\mathrm{p}$ values $<0.05$ were considered statistically significant. All analyses were conducted using SAS software V.9.4.

\section{RESULTS}

\section{Characteristics of the population at inclusion}

Compared with controls, a higher percentage of cases had no children (cases: 17.8\%; controls: 9.1\%) (table 1). Cases had a first full-term pregnancy at older ages (cases: 23 years; controls: 21 years), breast fed their children for shorter periods (breast feeding for at least 12 months: cases: $46.1 \%$; controls: $65.5 \%$ ), had more frequently a history of benign breast disease (cases: $36.4 \%$; controls: $12.6 \%$ ) and shorter duration of daily moderate intensity physical activity (cases: 2.0 hours; controls: 3.6 hours), and higher percentage completing secondary school (cases: 39.4\%; controls: $25.9 \%$ ) than controls. In addition, cases were taller ( 1.57 vs $1.56 \mathrm{~m}$ ), leaner (64 vs $68 \mathrm{~kg}$ ) and had lower BMI (26.1 vs $28.1 \mathrm{~kg} / \mathrm{m}^{2}$ ) compared with the controls. Among tumours with available IHC $(n=332)$, $72 \%$ were ER+, $69 \%$ were progesterone receptor positive, while $21 \%$ of the tumours were $\mathrm{TN}$.

\section{Energy intake}

Total caloric intake was slightly higher among cases than controls (cases: $2853 \mathrm{kcal} /$ day; controls: $2694 \mathrm{kcal} /$ day) as well as the caloric intake from ultra-processed foods (NOVA 4) (cases: $729 \mathrm{kcal} /$ day; controls: $641 \mathrm{kcal} /$ day) (table 1). However, differences were not statistically significant.

\section{Relative contribution of subgroups to ultra-processed food} consumption in diet

The food subgroups contributing to ultra-processed foods in our population overall and by countries are presented in figures 1 and 2. Overall the major contributors were ready-to-eat/heat foods $(18.2 \%)$, cakes and desserts $(16.7 \%)$, carbonated and industrial fruit juice beverages $(16.7 \%)$, breakfast cereals $(12.9 \%)$, sausages and reconstituted meat products $(12.1 \%)$, yoghurt and dairy-based drinks $(7.6 \%)$, industrial bread $(6.1 \%)$ and package savoury snacks $(6.1 \%)$. Some differences were observed between countries. In Costa Rica and Colombia the highest contributors were carbonated and industrial fruit juice beverage and ready-to-eat/heat foods while in Chile and Mexico ready-to-eat/heat foods and cakes and desserts were the most frequently eaten ultra-processed foods (figure 2).

Higher energy intake among cases than controls were observed for industrial fruit juice beverages $(p=0.0001)$, industrial bread $(\mathrm{p}=0.0001)$, breakfast cereals $(0.0001)$, packaged sweet snacks $(\mathrm{p}=0.0001)$, packaged savoury snacks $(\mathrm{p}=0.001)$ and margarine and butter substitute (0.006) (online supplemental table 1 ).

\section{Ultra-processed food intake and breast cancer}

In the multivariate conditional regression model (table 2, model 1), a positive association was observed between overall ultra-processed food intake and risk of breast cancer $\left(\mathrm{OR}_{\mathrm{T} 3 \mathrm{Tl}}=1.49,95 \% \mathrm{CI}=0.96\right.$ to 2.33 , P-trend $\left.=0.07\right)$. Further adjustment for energy intake from other NOVA groups (NOVA 1-NOVA 3) strengthened this association $\left(\mathrm{OR}_{\mathrm{T}-\mathrm{T} 1}=1.93,95 \% \mathrm{CI}=1.11\right.$ to 3.35 , P-trend $=0.02$, model 2). In stratified analyses by breast cancer subtypes, a positive association of ultra-processed foods intake and $\mathrm{ER}+$ breast cancer was observed $\left(\mathrm{OR}_{\mathrm{T} 3 \mathrm{~T} 1}=1.70\right.$, $\quad(95 \%$ $\mathrm{CI}=0.82$ to 3.52 , P-trend $=0.15$ model 1 and $\mathrm{OR}_{\mathrm{T}-\mathrm{T} 1}=2.44$, $95 \% \mathrm{CI}=1.01$ to 5.90 , P-trend=0.049 model 2) while among ER- breast tumours, the overall ultra-processed food intake was not associated with the risk of breast cancer $\left(\mathrm{OR}_{\mathrm{T}-\mathrm{T} 1}=1.28,95 \% \mathrm{CI}=0.44\right.$ to 3.72 , P-trend $=0.64$ model $1, \mathrm{OR}_{\mathrm{T} 3-\mathrm{T} 1}=1.87,95 \% \mathrm{CI}=0.43$ to 8.13 , P-trend $=0.36$ model 2). Similar results were observed when percentage of total calories from ultra-processed foods was used as exposure variable (online supplemental table 2).

We explored further the role of some specific subgroups of ultra-processed foods. The most strongly associated with the risk of breast cancer were: industrial fruit juice beverages $\left(\mathrm{OR}_{\mathrm{T} 3-\mathrm{T} 1}=2.97,95 \% \mathrm{CI}=2.01\right.$ to 4.38 , P-trend $<0.0001$ model 2); industrial breads $\left(\mathrm{OR}_{\mathrm{T} 3-\mathrm{Tl}}=1.98,95 \% \mathrm{CI}=1.33\right.$ to 2.94, P-trend=0.001 model 2), breakfast cereals $\left(\mathrm{OR}_{\mathrm{Tz}-}\right.$ ${ }_{\mathrm{T} 1}=1.73,95 \% \mathrm{CI}=1.17$ to 2.56 , P-trend $=0.007$ model 2 ), packaged sweet snacks $\left(\mathrm{OR}_{\mathrm{T}-\mathrm{T} 1}=1.75,95 \% \mathrm{CI}=1.22\right.$ to 2.50 , P-trend $=0.003$ model 2), cakes and desserts $\left(\mathrm{OR}_{\mathrm{T} 3-\mathrm{T} 1}=1.83\right.$, $95 \% \mathrm{CI}=1.20$ to 2.79 , P-trend $=0.005$ model 2 ) and readyto-eat/heat foods $\left(\mathrm{OR}_{\mathrm{T}-\mathrm{-T}}=1.97,95 \% \mathrm{CI}=1.30\right.$ to 2.99 , P-trend $=0.002$ model 2) (online supplemental table 3). Similar results were observed among ER + breast cancer. Among ER- breast cancer and TN breast cancer strong associations were observed with ready-to-eat/heat foods $\left(\mathrm{OR}_{\mathrm{T} 3-\mathrm{T} 1}=5.53,95 \% \mathrm{CI}=1.71\right.$ to 18.0 , P-trend $=0.003$ and $\mathrm{OR}_{\mathrm{T} 3-\mathrm{Tl}}=4.98,95 \% \mathrm{CI}=1.47$ to 16.8 , P-trend $=0.01$, respectively). Our results remained significant after correction for multiple comparisons.

Based on the proportion of calories intake from ultraprocessed foods and assuming a linear relationship, we calculated that an increase of $20 \%$ in calories from ultraprocessed foods was related to a $46 \%$ increase in the risk of breast cancer. Similar results were observed among ER + breast cancer, while no association was observed with ER- tumours risk.

Blood concentrations of total industrial trans-fatty acids resulting from industrial processing showed an increasing gradient ranging from $0.38 \%$ (Costa Rica) to $0.60 \%$ of total fatty acids (Mexico) and were marginally correlated with overall ultra-processed food intake $(\mathrm{r}=0.11 \mathrm{p}<0.08)$. This correlation increased to $\mathrm{r}=0.21 \quad(\mathrm{p}=0.0005)$ when we restricted the ultra-processed items including yoghurts and dairy drinks, industrial bread, confectionary, cakes 
Table 1 Main characteristics of the population

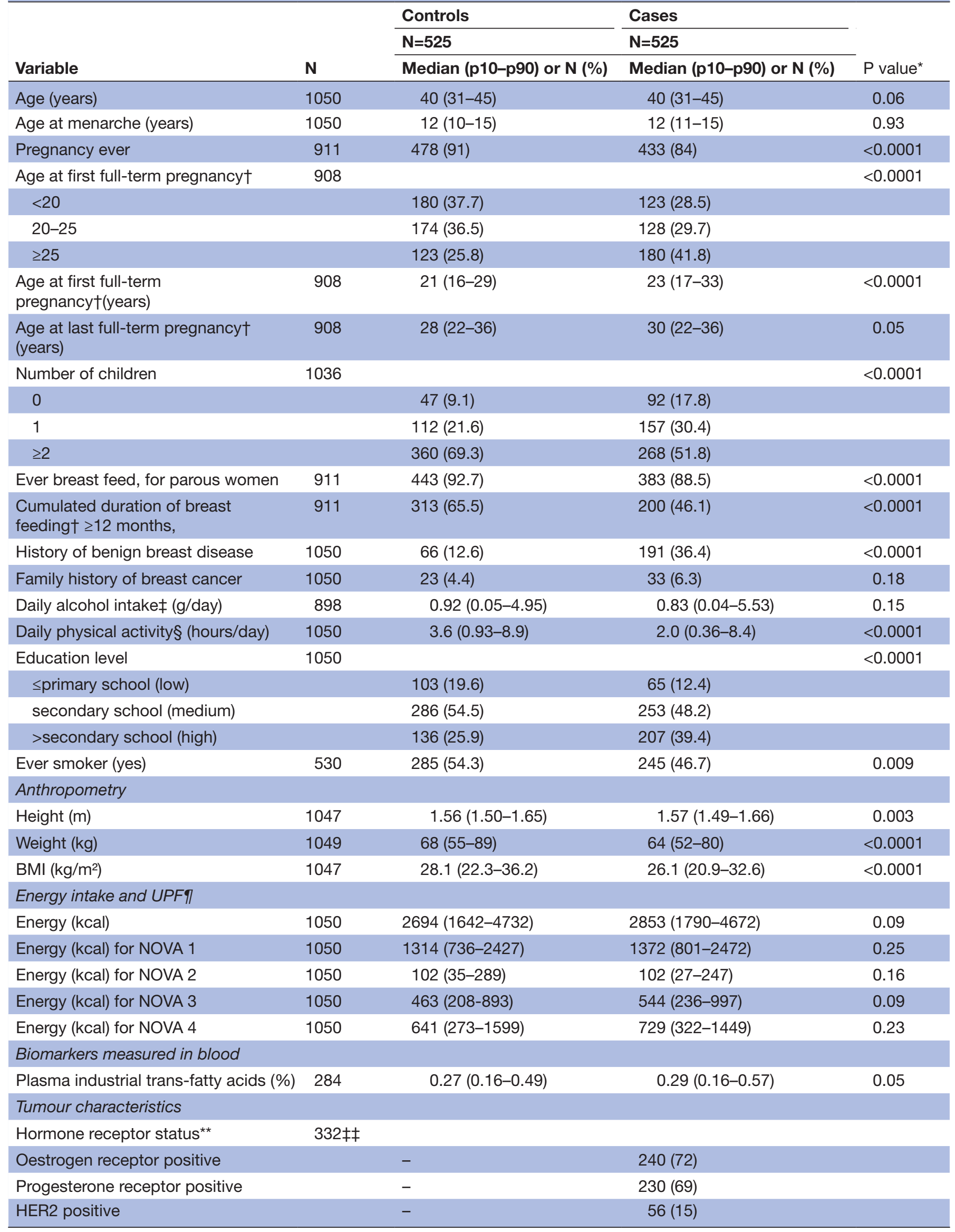




\begin{tabular}{|c|c|c|c|c|}
\hline \multirow[b]{3}{*}{ Variable } & \multirow[b]{3}{*}{$\mathbf{N}$} & Controls & Cases & \multirow[b]{3}{*}{$P$ value* } \\
\hline & & $\mathrm{N}=525$ & $\mathrm{~N}=525$ & \\
\hline & & Median (p10-p90) or N (\%) & Median (p10-p90) or N (\%) & \\
\hline Of which Basal-liket† & & - & $61(18)$ & \\
\hline
\end{tabular}

${ }^{*} \mathrm{P}$ value Wilcoxon test from logistic regression conditional on matching factors.

†Among parous women only.

$\ddagger$ Among consumers.

$\S$ Daily moderate intensity physical activity.

INOVA group 1: Unprocessed or minimally processed foods; NOVA group 2: Processed culinary ingredients, NOVA group 3:

Processed foods; NOVA group 4: Ultra-processed food and drink products.

**Immunohistochemistry is so far available for 332 cases. Percentages given for tumour characteristics are based on these 332 cases. Receptors status presented irrespective of other receptors.

††Basal-like: (TN and EGFR +and/or CK5/6+).

BMI, body mass index; CK5/6, cytokeratin 5/6; EGFR, epidermal growth factor receptor; ER, oestrogen receptor; HER2, human epidermal growth factor receptor 2; PR, progesterone receptor; TN, triple negative; UPF, ultra-processed foods.

and deserts, ready-to-eat/heat foods and margarine and butter substitutes. (online supplemental table 4)

\section{DISCUSSION}

In our population of young Latin American women, ultraprocessed food intake was associated with an increased risk of breast cancer. An increase of $20 \%$ in calories from ultra-processed foods was related to a $46 \%$ increase in the risk of breast cancer. Similar results were observed among $\mathrm{ER}+$ breast cancer, while no association was observed with ER- tumours risk. The food groups most strongly associated to the risk of breast cancer were industrial fruit juices, industrial bread, packaged sweet snacks and readyto-eat/heat foods.

This is the first study on the association of ultraprocessed foods intake and breast cancer in young women from Latin American countries where a rapid nutritional transition is taking place. ${ }^{3}$ Ultra-processed foods are usually associated with high energy density, high intake of sugar, low levels of micronutrients, greater content of
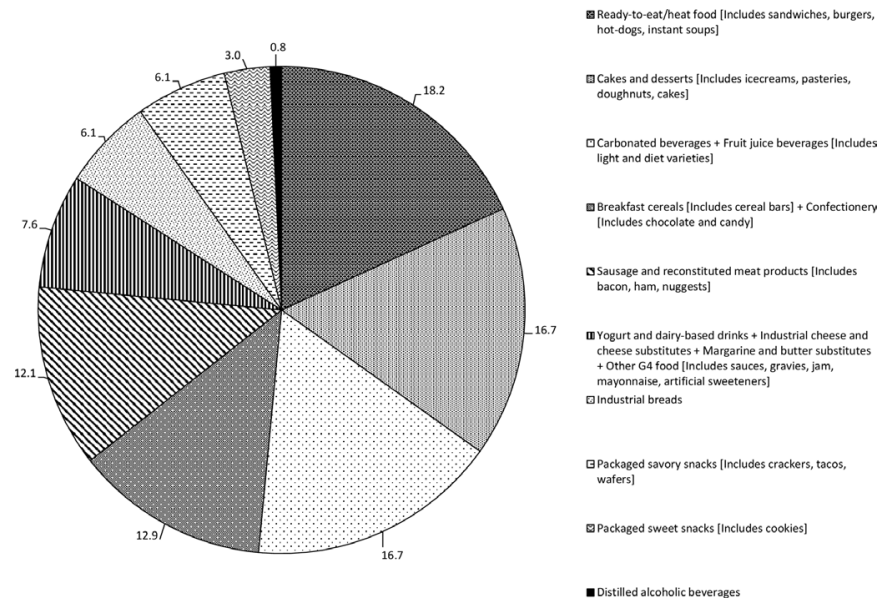

Figure 1 Food groups contributing to ultra-processed foods in our population overall. unhealthy fats and lower content of protein and fibre. ${ }^{19}$ Previous studies have shown an association with increased risk of chronic diseases in particular obesity, ${ }^{8021}$ hypertension, ${ }^{22}$ metabolic syndrome and dyslipidaemia ${ }^{823}$ and cardiovascular and cerebrovascular diseases. ${ }^{24}$

Recently a large prospective study has reported a significant association of ultra-processed food intake and cancer incidence in the French population. ${ }^{11}$ In this study, the contribution of ultra-processed foods to the overall diet in women was $18.7 \%$ while in our population, the contribution was $23.7 \%$ among the controls and $25.5 \%$ among the cases. An association of ultra-processed food intake was observed on overall cancers as well as postmenopausal breast cancer but no effect was observed among premenopausal breast cancer. This may be due to the small number of premenopausal breast cancers $(n=264)$ in this study. ${ }^{11}$

Several potential mechanisms might explain our findings. Ultra-processed foods have been associated with a
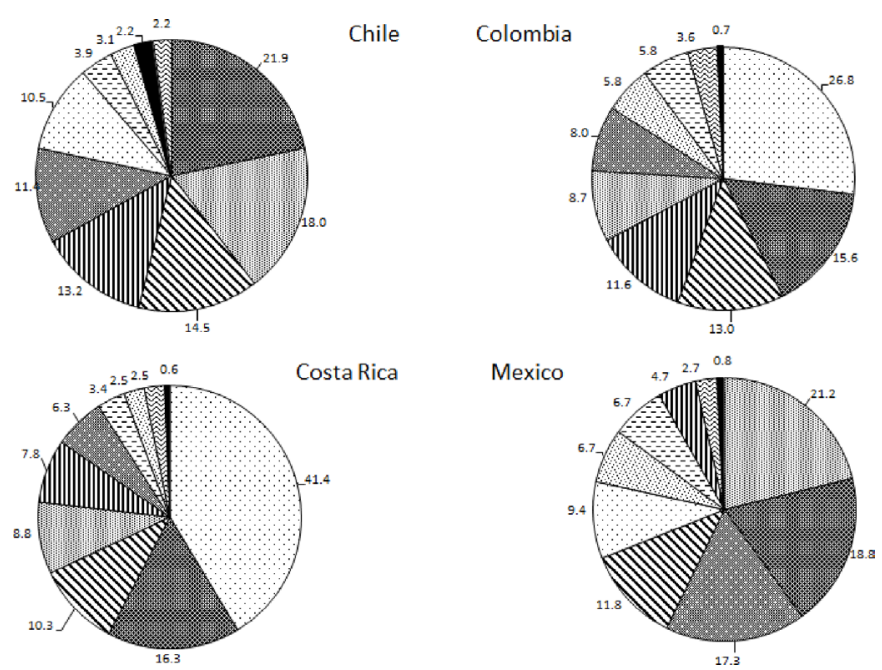

Figure 2 Food groups contributing to ultra-processed foods in our population by countries (Chile, Colombia, Costa Rica and Mexico). 
Table 2 ORs and 95\% Cl for associations between ultra-processed food intake and risk of breast cancer, overall and by receptor status

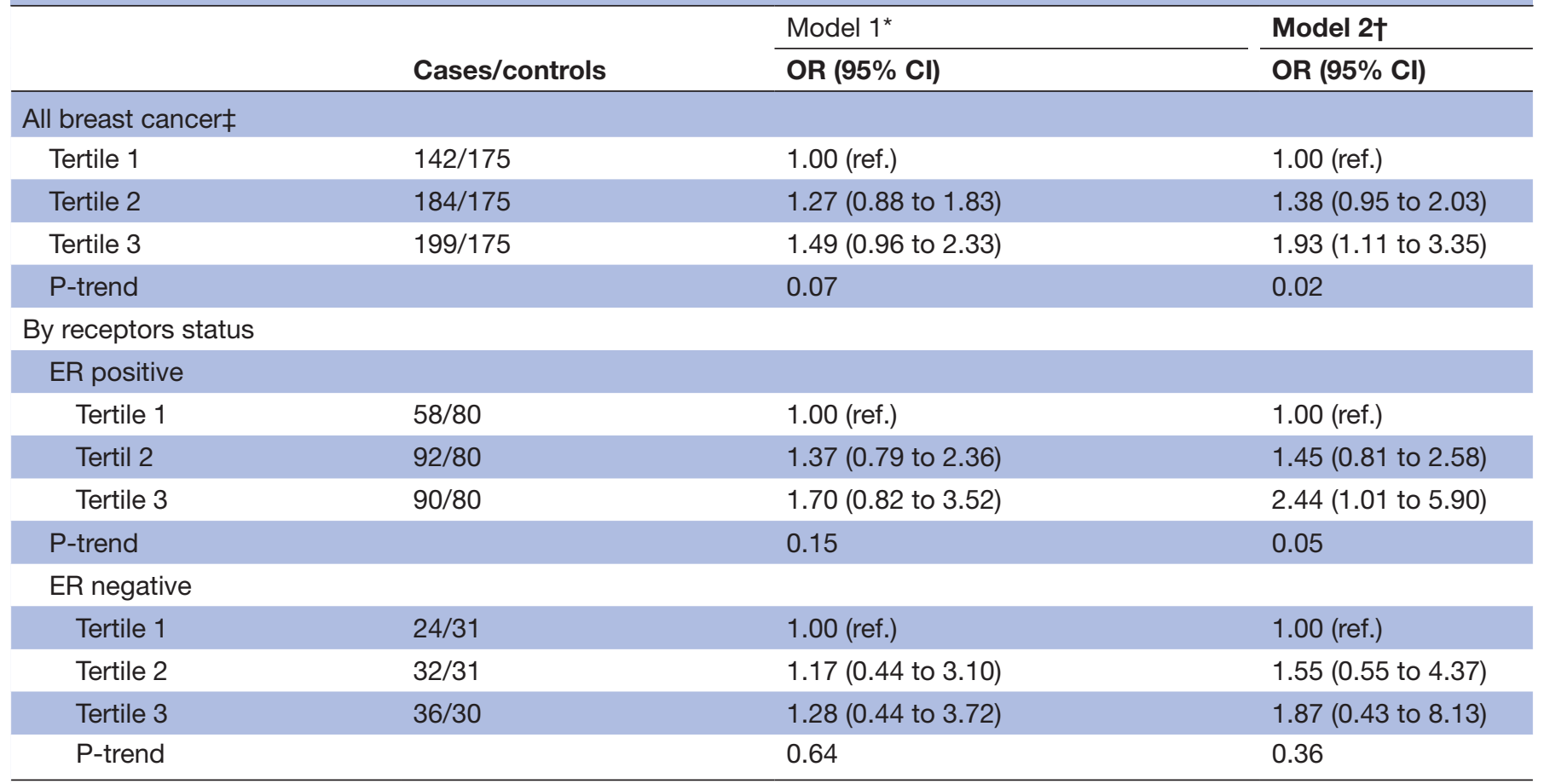

*Model 1: ORs were estimated by logistic regression conditioned on age $( \pm 3$ years), city district of residence and health insurance institution and adjusted for education, (sprimary/secondary/>secondary), moderate intensity physical activity (continuous), number of full-term pregnancies (continuous), age at first full-term pregnancy (nulliparous/<20; 20-25; $\geq 25$ ), breast feeding ever (yes/no), BMI (continuous) and total energy intake (continuous).

†Model 2: Additionally adjusted for energy intake from the other NOVA groups (NOVA 1, NOVA 2, NOVA 3 added simultaneously in the model).

$\ddagger$ Cut-off points for tertiles are respectively: Tertile $1=\leq 495.5$; tertile $2=495.5-896.6$; tertile $3=>896.6 \mathrm{kcal} /$ day.

$\mathrm{BMI}$, body mass index; ER, oestrogen receptor .

higher glycaemic response and lower satiety effect. ${ }^{8} \mathrm{~A}$ recent meta-analysis of prospective studies reported a significant positive association between glycaemic index and glycaemic load and breast cancer. ${ }^{25}$ Supporting this hypothesis, high intake of carbohydrate has been linked to a higher risk of breast cancer among Mexican women. ${ }^{26}$ The strong association with industrial fruit juice and packaged sweet snacks in our study supports this hypothesis. Other dietary factors may be involved as a combination of low fat and high fibre intake has been associated to a significant reduction of breast cancer in premenopausal women. ${ }^{27}$

Ultra-processed products are also rich in fat and industrially transformed fat, in particular trans-fatty acids formed from fats and oil by partial hydrogenation during industrial processing techniques. A large cohort study conducted in European countries (European Prospective Investigation into Cancer and Nutrition (EPIC)) has shown an increase in breast cancer risk in association with blood industrial trans-fatty acids in particular for ER- breast cancer. ${ }^{10}$ These fatty acids are found in fast food, and EPIC levels measured in blood have been used as biomarkers of dietary intake of industrial trans fats. ${ }^{28}$ In the present study, ultraprocessed food intake was significantly correlated to the blood levels of total industrial trans-fatty acids. This may suggest that ultra-processed foods, through their content in trans fat, could affect the risk of breast cancer in premenopausal women.

Little research has examined whether ultraprocessed foods have effects on health independent of their nutrient content. Several studies focusing on obesity have observed that the association between ultra-processed food intake and obesity persists after accounting for nutrient intake (eg, saturated fat, trans fat, added sugar and fibre intake, sodium) suggesting that ultra-processed foods may promote additional adverse health outcomes independent of nutrient content. ${ }^{8}$ The wide range of additives that are added to ultra-processed foods as well as other products such acrylamide produced during heat treatment or contaminants migrating from plastic containers such as bisphenol $\mathrm{A}$ and phthalate which have endocrine disruptors properties could also play a role but no firm data are currently available. ${ }^{29} 30$

Our study is the largest ongoing multicentric population-based case-control study of premenopausal breast cancer in Latin America. Strengths include the study of young women with large variety and wide range of exposure in dietary intake, the use of standardised 
methodology to collected dietary data across centres using validated semi-quantitative food frequency questionnaires and standardised methods for food items classification regarding processing classification with nutritional experts, the use of standardised tools and questionnaires across centres to collect information on various current and past lifestyle factors and the centralisation of IHC analyses, thus limiting the interlaboratory variability. Still some limitations need to be acknowledged. We used a case-control design in order to assemble primary incident breast cancer cases in young women and recall of exposure may be an issue. However, participants were not aware of the hypothesis of the study and had limited knowledge on dietary factor associated to breast cancer. While food frequency questionnaires provide less detailed information on food processing than data from 24 hours recalls, our questionnaire included a list of specific ready-to-eat/heat foods and type of food consumed and mainly aimed at assessing usual dietary intakes in the few years before breast cancer diagnosis (which cannot be obtained from 24 hours dietary recalls). It should be acknowledged that the few years prior to breast cancer diagnosis might not represent the exposure relevant for breast cancer development; however, several studies have shown that adult diet tends to track overtime. ${ }^{31}$ For some products, the food processing techniques might have changed. Thus, some misclassification could be expected. This would most likely be a random misclassification that would tend to lower the observed associations. Finally, our models were adjusted for potential confounding factors, total caloric intake and dietary intake from other NOVA groups, though potential residual confounding is still possible given that ultra-processed food intake is usually associated with unhealthy lifestyle. In addition, the modest sample size for ER- and TN tumours limits the interpretation of the results for these breast cancer subtypes.

Our results support an adverse effect of ultraprocessed food intake on the risk of breast cancer in young Latin American women. Further studies are needed to confirm our results and disentangle mechanistic pathways. Given the already proven chronic adverse health effects of ultra-processed foods and the modifiable nature of the diet, a decrease in ultraprocessed foods intake should be encouraged particularly in Latin America where an increasing trend of ultra-processed food intake is observed.

\footnotetext{
Author affiliations

${ }^{1}$ Centre for Population Health Research, National Institute of Public Health, Cuernavaca, Morelos, Mexico

${ }^{2}$ Hubert Department of Global Health, Emory University, Atlanta, Georgia, USA ${ }^{3}$ Department of Nutrition, Faculty of Public Health, University of Sao Paulo, Sao Paulo, Brazil

${ }^{4}$ Harvard University T. H. Chan School of Public Health, Boston, Massachusetts, USA ${ }^{5}$ Nutrition and Metabolism Branch, International Agency for Research on Cancer (IARC/WHO), Lyon, France
}

${ }^{6}$ Servicio de Patología del Hospital de Oncología, Centro Médico Nacional Siglo XXI, Instituto Mexicano del Seguro Social, Ciudad de Mexico, Mexico

${ }^{7}$ Group Infection and Cancer, School of Medicine, University of Antioquia, Medellín, Colombia

${ }^{8}$ Grupo Impacto de los Componentes Alimentarios en la Salud, School of Nutrition and Dietetics, University of Antioquia, Medellín, Colombia

${ }^{9}$ Agencia Costarricense de Investigaciones Biomédicas (ACIB) - Fundación

INCIENSA, San Jose, Costa Rica

${ }^{10}$ Instituto de Nutrición y de Tecnología de los Alimentos, Universidad de Chile,

Santiago de Chile, Chile

${ }^{11}$ Division of Human Biology, Fred Hutchinson Cancer Research Center, Seattle, Washington, USA

Acknowledgements The authors wish to thank the substantial support provided by the research nurses and health workers as well as Tracy Lignini, Dacia Christin, Cecile Le Duc, Jordi de Battle, Talita Duarte-Salles and Ana Cristina Ocampo for the logistical and data basis development. The authors also wish to thank the women participating in the project, for their time and commitment.

Contributors IR, SR, GTM, GIS, CP, ACRo, MLG and PLP designed the study. GTM, AA-L, IA-C, GIS, MEM, CP, ACRo and MLG and members of the PRECAMA team (JT, EN, RJ, YRC, AA, CAO, WHA, GB, ACRa, CE, FH, IDY, AH, BC, PG, RO, DG, VL, LM and JLS) collected the data and sample materials. PLP designed the specific protocols for IHC and genetic analyses. PLP and MG, together with members of the PRECAMA team (ED, JG, TD, KW and HL), conducted molecular analyses. IR, IH, AK and NK developed the dietary data basis and NOVA classification together with members of the PRECAMA team (MLG, GTM, GIS and CP). VC developed the laboratory assay for fatty acids measurements, and VC and EKA conducted the analyses. CB assembled the database and analysed the data. IR, SR, NK, IH, GTM, GIS, CP, ACRo, MLG, MH, VC and MLG interpreted the data. All authors participated in writing the manuscript. All authors read and approved the final manuscript. SR is the guarantor for the study

Funding The study is funded by the International Agency for Research on Cancer (IARC), the Union for International Cancer Control (UICC), the Pan American Health Organization (PAHO), the Ibero-American Programme for the Development of Science and Technology (CYTED), Ministerio de Ciencia, Tecnología e Innovación (MINCIENCIAS, grant \#111584267659). The funders had no role in study design, data collection and analysis, decision to publish or preparation of the manuscript. ACR was part of the Proyecto Guanacaste when this work was carried out.

Disclaimer Where authors are identified as personnel of the International Agency for Research on Cancer/WHO, the authors alone are responsible for the views expressed in this article and they do not necessarily represent the decisions, policy or views of the International Agency for Research on Cancer/WHO.

Competing interests None declared.

\section{Patient consent for publication Not applicable.}

Ethics approval All participants gave written informed consent before enrolment, and the study protocols were approved by the institutional review boards of Chile (Oncologic Institute Foundation Arturo Lopez Pérez, Instituto de Nutrición y de Tecnología de Ios Alimentos, National Cancer Institute), Colombia (Cancer Institute Las Americas and University of Antioquia), Costa Rica (Costa Rican Institute of Clinical Research (ICIC) and Center for Strategic Development and Information in Health and Social Security (CENDEISSS) of the Costa Rican Social Security Fund (CCSS)), Mexico (National Institute of Public Health and the Social Security Mexican Institute) and the International Agency for Research on Cancer (IARC). All methods were performed in accordance with the relevant guidelines and regulations of these approvals.

Provenance and peer review Not commissioned; externally peer reviewed.

Data availability statement Data are available upon reasonable request. PRECAMA data and biospecimens are available for investigators who seek to answer important questions on health and disease in the context of research projects that are consistent with the legal and ethical standard practices of IARC/ WHO and the PRECAMA Centres. The primary responsibility for accessing the data belongs to the PRECAMA centres that provided them. The use of anonymised data from the PRECAMA study can be requested by contacting the corresponding author. The request will then be passed to members of the PRECAMA Steering Committee for deliberation.

Supplemental material This content has been supplied by the author(s). It has not been vetted by BMJ Publishing Group Limited (BMJ) and may not have been 
peer-reviewed. Any opinions or recommendations discussed are solely those of the author(s) and are not endorsed by BMJ. BMJ disclaims all liability and responsibility arising from any reliance placed on the content. Where the content includes any translated material, BMJ does not warrant the accuracy and reliability of the translations (including but not limited to local regulations, clinical guidelines, terminology, drug names and drug dosages), and is not responsible for any error and/or omissions arising from translation and adaptation or otherwise.

Open access This is an open access article distributed in accordance with the Creative Commons Attribution Non Commercial (CC BY-NC 4.0) license, which permits others to distribute, remix, adapt, build upon this work non-commercially, and license their derivative works on different terms, provided the original work is properly cited, appropriate credit is given, any changes made indicated, and the use is non-commercial. See: http://creativecommons.org/licenses/by-nc/4.0/.

\section{ORCID iD}

Sabina Rinaldi http://orcid.org/0000-0002-6846-1204

\section{REFERENCES}

1 Villarreal-Garza C, Aguila C, Magallanes-Hoyos MC, et al. Breast cancer in young women in Latin America: an unmet, growing burden. Oncologist 2013;18 Suppl:26-34.

2 Colditz GA, Rosner BA, Chen WY, et al. Risk factors for breast cancer according to estrogen and progesterone receptor status. $J$ Natl Cancer Inst 2004;96:218-28.

3 Santiago-Torres M, Tinker LF, Allison MA, et al. Development and use of a traditional Mexican diet score in relation to systemic inflammation and insulin resistance among women of Mexican descent. J Nutr 2015;145:2732-40.

4 Sánchez-Zamorano LM, Flores-Luna L, Ángeles-Llerenas A, et al. Healthy lifestyle on the risk of breast cancer. Cancer Epidemiol Biomarkers Prev 2011;20:912-22.

5 Monteiro CA, Cannon G, Levy RB, et al. Ultra-processed foods: what they are and how to identify them. Public Health Nutr 2019;22:936-41.

6 Monteiro CA, Cannon G, Lawrence M, et al. Ultra-processed foods, diet quality, and health using the nova classification system. Rome: FAO, 2019

7 Moubarac JC. Ultra-processed food and drink products in Latin America: trends, impact on obesity, policy implications. Washington D.C: PAHO, 2015.

8 Poti JM, Braga B, Qin B. Ultra-processed food intake and obesity: what really matters for Health-Processing or nutrient content? Curr Obes Rep 2017;6:420-31.

9 Bray F, Ferlay J, Soerjomataram I, et al. Global cancer statistics 2018: GLOBOCAN estimates of incidence and mortality worldwide for 36 cancers in 185 countries. CA Cancer J Clin 2018;68:394-424.

10 Chajès $\mathrm{V}$, Assi $\mathrm{N}$, Biessy $\mathrm{C}$, et al. A prospective evaluation of plasma phospholipid fatty acids and breast cancer risk in the EPIC study. Ann Oncol 2017;28:2836-42.

11 Fiolet T, Srour B, Sellem L, et al. Consumption of ultra-processed foods and cancer risk: results from NutriNet-Santé prospective cohort. BMJ 2018;360:k322.

12 Romieu I, Biessy C, Torres-Mejía G, et al. Project profile: a multicenter study on breast cancer in young women in Latin America (PRECAMA study). Salud Publica Mex 2019;61:601-8.
13 Lohman TG RA, Martorell R. Anthropometric standardization reference manual, 1988.

14 Romieu I, Parra S, Hernández JF, et al. Questionnaire assessment of antioxidants and retinol intakes in Mexican women. Arch Med Res 1999;30:224-39.

15 Hernández-Avila M, Romieu I, Parra S, et al. Validity and reproducibility of a food frequency questionnaire to assess dietary intake of women living in Mexico City. Salud Publica Mex 1998;40:133-40.

16 Kabagambe EK, Baylin A, Allan DA, et al. Application of the method of triads to evaluate the performance of food frequency questionnaires and biomarkers as indicators of long-term dietary intake. Am J Epidemiol 2001;154:1126-35.

17 Hogg R, Tanis E. Probability and statistical inference, 2006.

18 Hochberg Y, Benjamini Y. More powerful procedures for multiple significance testing. Stat Med 1990;9:811-8.

19 Monteiro CA, Cannon G, Moubarac JC, et al. Ultra-processing. An odd 'appraisal'. Public Health Nutr 2018;21:497-501.

20 Mendonça RdeD, Pimenta AM, Gea A, et al. Ultraprocessed food consumption and risk of overweight and obesity: the University of Navarra follow-up (SUN) cohort study. Am J Clin Nutr 2016;104:1433-40.

21 Vandevijvere S, Jaacks LM, Monteiro CA, et al. Global trends in ultraprocessed food and drink product sales and their association with adult body mass index trajectories. Obes Rev 2019;20 Suppl 2:10-19.

22 Mendonça RdeD, Lopes ACS, Pimenta AM, et al. Ultra-Processed food consumption and the incidence of hypertension in a Mediterranean cohort: the Seguimiento Universidad de Navarra project. Am J Hypertens 2017;30:358-66.

23 Kelly B, Jacoby E. Public health nutrition special issue on ultraprocessed foods. Public Health Nutr 2018;21:1-4.

24 Srour B, Fezeu LK, Kesse-Guyot E, et al. Ultra-processed food intake and risk of cardiovascular disease: prospective cohort study (NutriNet-Santé). BMJ 2019;365:I1451.

25 Choi Y, Giovannucci E, Lee JE. Glycaemic index and glycaemic load in relation to risk of diabetes-related cancers: a meta-analysis. $\mathrm{Br} \mathrm{J}$ Nutr 2012;108:1934-47.

26 Romieu I, Lazcano-Ponce E, Sanchez-Zamorano LM, et al. Carbohydrates and the risk of breast cancer among Mexican women. Cancer Epidemiol Biomarkers Prev 2004;13:1283-9.

27 Ferrari P, Rinaldi S, Jenab M, et al. Dietary fiber intake and risk of hormonal receptor-defined breast cancer in the European prospective investigation into cancer and nutrition study. Am J Clin Nutr 2013;97:344-53.

28 Chajès V, Biessy C, Byrnes G, et al. Ecological-level associations between highly processed food intakes and plasma phospholipid elaidic acid concentrations: results from a cross-sectional study within the European prospective investigation into cancer and nutrition (EPIC). Nutr Cancer 2011;63:1235-50.

29 Burks H, Pashos N, Martin E, et al. Endocrine disruptors and the tumor microenvironment: a new paradigm in breast cancer biology. Mol Cell Endocrinol 2017;457:13-19.

30 Ahern TP, Broe A, Lash TL, et al. Phthalate exposure and breast cancer incidence: a Danish nationwide cohort study. J Clin Oncol 2019;37:1800-9. +

31 Willett W. Nutritional epidemiology. 3rd edn. Oxford: Oxford University Press, 2013 